\title{
On Distance-Regular Graphs in Which Neighborhoods of Vertices Are Strongly Regular
}

\author{
A. L. Gavrilyuk ${ }^{a}$, Corresponding Member of the RAS A. A. Makhnev ${ }^{a, b}$, and D. V. Paduchikh \\ Received February 27, 2013
}

DOI: $10.1134 / \mathrm{S} 1064562413050116$

We consider undirected graphs without loops or multiple edges. Given a vertex $a$ in a graph $\Gamma$, let $\Gamma_{i}(a)$ denote the $i$-neighborhood of $a$, i.e., the subgraph induced by $\Gamma$ on the set of all its vertices that are a distance of $i$ away from $a$. Let $[a]=\Gamma_{1}(a)$ and $a^{\perp}=\{a\} \cup[a]$.

A strongly regular graph with parameters $(v, k, \lambda, \mu)$ and with integer eigenvalues $k, r$, and $s$, where $s<0$, is called a Smith graph if

$$
\begin{gathered}
k=\frac{-s((2 r+1)(r-s)-r(r+1))}{(r-s)+r(r+1)}, \\
l=\frac{-(s+1)((2 r+1(r-s)-r(r+1))}{(r-s)-r(r+1)}, \\
\lambda=\frac{-r(s+1)((r-s)-r(r+3))}{(r-s)+r(r+1)} \\
\mu=\frac{-(r+1) s((r-s)-r(r+1))}{(r-s)+r(r+1)} .
\end{gathered}
$$

If vertices $u$ and $w$ are separated by a distance of $i$ in $\Gamma$, then $b_{i}(u, w)\left(c_{i}(u, w)\right)$ denotes the number of vertices in the intersection of $\Gamma_{i+1}(u)\left(\Gamma_{i-1}(u)\right)$ with [w]. A graph $\Gamma$ of diameter $d$ is called a distance-regular graph with the intersection array $\left\{b_{0}, b_{1}, \ldots, b_{d-1} ; c_{1}\right.$, $\left.c_{2}, \ldots, c_{d}\right\}$ if the values $b_{i}(u, w)$ and $c_{i}(u, w)$ are independent of the choice of the vertices $u$ and $w$ separated by a distance of $i$ in $\Gamma$ for any $i=0,1, \ldots, d$. Let $a_{i}=k-$ $b_{i}-c_{i}$. The Taylor graph is a distance-regular graph with the intersection array $\{k, \mu, 1 ; 1, \mu, k\}$ (see [1]).

Let $\Gamma$ be a distance-regular graph of diameter $d \geq 3$, and let $\theta_{0}>\theta_{1}>\ldots>\theta_{d}$ be the eigenvalues of $\Gamma$. According to [2], we have the fundamental bound

$$
\left(\theta_{1}+\frac{k}{a_{1}+1}\right)\left(\theta_{d}+\frac{k}{a_{1}+1}\right) \geq-\frac{k a_{1} b_{1}}{\left(a_{1}+1\right)^{2}} .
$$

\footnotetext{
${ }^{a}$ Krasovskii Institute of Mathematics and Mechanics, Ural Branch, Russian Academy of Sciences, ul. S. Kovalevskoi 16, Yekaterinburg, 620219 Russia

${ }^{b}$ Ural Federal University, ul. Mira 19, Yekaterinburg, 620002 Russia e-mail: alexander.gavriliouk@gmail.com, makhnev@imm.uran.ru
}

Let

$$
b^{+}=-1-\frac{b_{1}}{1+\theta_{d}}, \quad b^{-}=-1-\frac{b_{1}}{1+1+\theta_{1}} .
$$

A nonbipartite graph for which the fundamental bound becomes an equality is called tight. The neighborhood of any vertex in a tight graph is a strongly regular graph with eigenvalues $a_{1}, b^{+}, b^{-}$. It is well known that a tight graph of diameter 3 is a Taylor graph (see, e.g., [2, Theorem 3.2]). In this case, the neighborhood of any vertex is a strongly regular graph with $k^{\prime}=2 \mu^{\prime}$.

An incidence system with a set of points $P$ and a set of straight lines $\mathscr{L}$ is called an $\alpha$-partial geometry of order $(s, t)$ (denoted by $\left.p G_{\alpha}(s, t)\right)$ if each line contains $s+1$ points; each point lies on $t+1$ lines; any two points lie on at most one line; and, for any antiflag $(a, l) \in(P, \mathscr{L})$, there are precisely $\alpha$ lines that pass through $a$ and intersect $l$. If $\alpha=1$, the geometry is called a generalized quadrangle and is denoted by $G Q(s, t)$. The point graph of a geometry is defined on the set of points $P$, and two points are adjacent if they lie on a line. The point graph of $p G_{\alpha}(s, t)$ is strongly regular with $v=(s+1)\left(1+\frac{s t}{\alpha}\right), k=s(t+1), \lambda=s-$ $1+t(\alpha-1)$, and $\mu=\alpha(t+1)$. A strongly regular graph with such parameters for some positive integers $\alpha, s$, and $t$ is called a pseudogeometric graph for $p G_{\alpha}(s, t)$.

Makhnev has proposed a program for the study of distance-regular graphs in which the neighborhoods of vertices are strongly regular graphs with given parameters. This program has been implemented in the case of strongly regular graphs with the eigenvalue 2 (see [3]).

An antipodal distance-regular graph $\Gamma$ of diameter 3 has (see [1]) the intersection array $\{k, \mu(r-1), 1 ; 1, \mu, k\}$ and possesses $V=r(k+1)$ vertices and the spectrum $k^{1}, n^{f},(-1)^{k},(-m)^{h}$, where $n$ and $-m$ are the roots of the equation $x^{2}+(\mu-\lambda) x-k=0, f=$ $\frac{m(r-1)(k+1)}{n+m}$, and $h=\frac{n(r-1)(k+1)}{n+m}$. 
If $\mu \neq \lambda$, then the eigenvalues of the graph are integer and the parameters of the graph can be expressed in terms of $r, n$, and $m: k=n m, \mu=\frac{(m-1)(n+1)}{r}$, and $\lambda=\mu+n-m$. Since the multiplicities of the eigenvalues are integer-valued, we have the following divisibility condition: $n+m$ divides $(r-1) m\left(m^{2}-1\right)$. If $r>2$, the Krein condition $q_{33}^{3} \geq 0$ implies $m \leq n^{2}$. If $m=n^{2}$, then the neighborhood of any vertex is a strongly regular graph with eigenvalues $a_{1}=(n-$ 1) $\left(\frac{(n+1)^{2}}{r}-n\right), n-\frac{n+1}{r}$, and $n-\frac{(n+1)^{2}}{r}$. A graph with $m=n^{2}$ (equivalently, with $q_{33}^{3}=0$ ) is called a Krein graph of diameter 3.

Theorem 1. Let $n+1=r u$ and $\Gamma$ be a Krein graph of diameter 3 with the intersection array $\left\{n^{3},(r-1) u\left(n^{2}-1\right)\right.$, $\left.1 ; 1, u\left(n^{2}-1\right), n^{3}\right\}$. Then the following assertions hold:

(1) For any vertex $a \in \Gamma$, the subgraph $\Delta=[a]$ is a pseudogeometric graph for $p G_{u-1}\left(r u-2, r u^{2}-r u\right)$ with eigenvalues $(r u-2)\left(r u^{2}-r u+1\right), r u-1-u$, and $-\left(r u^{2}-r u+1\right)$.

(2) If $n$ is a power of a prime number, then there exist graphs $\Gamma$ and $\Delta$. Specifically,

(i) If $r=\frac{n+1}{2}$, then there exists a graph $\Gamma$ that is locally a $G Q(n-1, n+1)$-graph.

(ii) There exist pseudogeometric graphs for $p G_{2}(4$, 12), $p G_{3}(6,24), p G_{2}(7,18), p G_{4}(8,40), p G_{2}(10,24)$, $p G_{3}(10,36), p G_{5}(10,60)$, and $p G_{6}(12,84)$.

Let $\Gamma$ be an antipodal graph of diameter 4 and $\bar{\Gamma}$ be the antipodal quotient of $\Gamma$. Then, by [1, Proposition 4.2.2], $\Gamma$ has the intersection array $\left\{k, k-a_{1}-1\right.$, $\left.(r-1) c_{2}, 1 ; 1, c_{2}, k-a_{1}-1, k\right\}$. If the Krein parameters vanish, we have the following three interesting cases: $q_{11}^{4}=0, q_{44}^{4}=0$, and $q_{11}^{4}=q_{44}^{4}=0$.

The equality $q_{44}^{4}=0$ implies that $\bar{q}_{22}^{2}=0$ in $\bar{\Gamma}$ and $\bar{\Gamma}$ is a Smith graph. Therefore, the neighborhoods of vertices in $\Gamma$ (which are isomorphic to the neighborhoods of vertices in $\bar{\Gamma}$ ) are strongly regular. A graph with $q_{44}^{4}=0$ is called a Krein graph of diameter 4 . The following result concerning Krein graphs of diameter 4 is due to Jurisic [4].

Proposition 1. Let $\Gamma$ be a Krein graph of diameter 4 . Then one of the following assertions holds:

(1) $\bar{\Gamma}$ is a Smith graph without triangles, and $\Gamma$ has the intersection array $\{k, k-\mu,(r-1) \mu, 1 ; 1, \mu, k-1$, $k\}$, where $k=t^{3}+3 t^{2}+t$ and $r \mu=t(t+1)$.

(2) $\bar{\Gamma}$ is a Smith graph with $a_{1}>0$ and nonprincipal eigenvalues $t$ and $s$; and the nonprincipal eigenvalues of the constituents of $\bar{\Gamma}$ are equal to $t, s_{1}=\frac{t^{2}+2 t+s}{2}, t$, $s_{2}=\frac{s-t^{2}}{2}$ and either

(i) $t=q-2$ and $\Gamma$ is a tight graph $\mathrm{AT} 4(q-2, q, r)$ with the intersection array $\left\{q\left(q^{2}-2\right),\left(q^{2}-1\right)(q-1)\right.$, $\frac{(r-1) \cdot 2 q(q-1)}{r}, 1 ; 1, \frac{2 q(q-1)}{r},\left(q^{2}-1\right)(q-1)$, $\left.q\left(q^{2}-2\right)\right\}$

(ii) $\Gamma$ is a cover of the point graph of the generalized quadrangle $G Q\left(w, w^{2}\right), w=t+1$ is odd, and $4 w^{3}+$ $(w+1)^{2}$ is a square; or

(iii) $s$ and t are even, $q=t+2$ and $u=\frac{s+q^{2}}{2}$ satisfy $\sqrt{q} \leq u<q-1, q(q-1)-u$ divides $(q-2)^{2}(q-1)^{2} q$, and the expression $4 u^{4}(q-2)^{2}-4 u^{3}(q-1)\left(q^{3}-q^{2}-\right.$ $6 q+10)+u^{2}(q-1)^{2}\left(q^{4}+6 q^{3}-15 q^{2}+12 q+20\right)-$ $2 u(q-1)^{3}\left(q(q+1)\left(q^{2}+4\right)\right)+q^{2}(q-1)^{4}(q+2)^{2}$ is a square.

We show that case (2)(ii) is not possible. Let $w=$ $2 l+1$. Then $4 w^{3}+(w+1)^{2}=4\left(8 l^{3}+12 l^{2}+6 l+1\right)+$ $4\left(l^{2}+2 l+1\right)$. Therefore, $8 l^{3}+13 l^{2}+8 l+2$ is a square. If $l$ is odd, then $8 l^{3}+13 l^{2}+8 l+2$ is congruent to 3 modulo 4 . If $l$ is even, then $8 \beta+13 l^{2}+8 l+2$ is congruent to 2 modulo 4 . In either case, we obtain a contradiction.

By Theorem 5.2 in [2], $\Gamma$ is tight if and only if $q_{11}^{4}=0$. If $\Gamma$ is a tight graph with a neighborhood of a vertex having nonprincipal eigenvalues $p=b^{+},-q=b^{-}$, then all the parameters of $\Gamma$ can be expressed in terms of $p, q$, and $r$. In this case, $\Gamma$ is said to be an antipodal tight graph of diameter 4 with parameters $p, q, r$ (AT4 $(p, q, r)$-graph). The results obtained by Koolen and Jurisic [2,5] for AT4-graphs are stated as the following proposition.

Proposition 2. Let $\Gamma$ be an $\operatorname{AT} 4(p, q, r)$-graph. Then $\Gamma$ is a graph with the intersection array $\{q(p q+p+q)$, $\left(q^{2}-1\right)(p+1), \frac{(r-1) q(p+q)}{r}, 1 ; 1, \frac{q(p+q)}{r},\left(q^{2}-\right.$ 1) $(p+1), q(p q+p+q)\}$ and the following assertions hold:

(1) If $p=1$ or $r=p+q$, then $\Gamma$ is a Conway-Smith graph with $(p, q, r)=(1,2,3)$. 
(2) If $q=2$, then $\Gamma$ is a Johnson graph $J(8,4)$ with ( $p$, $q, r)=(2,2,2)$, a half-8-cube with $(p, q, r)=(4,2,2)$, or a Conway-Smith graph.

(3) If $(p, q, r)=(q s, q, q)$, then $\Gamma$ is a Johnson graph $J(8,4)$, a half-8-cube, a $3 O_{6}^{-}(3)$-graph with $(p, q, r)=$ $(3,3,3)$, the second Meixner graph with $(p, q, r)=(8,4$, $4)$, or a $3 O_{7}(3)$-graph with $(p, q, r)=(9,3,3)$.

The central result in this work is the following one, which was obtained by Makhnev.

Theorem 2. There do not exist $\operatorname{AT} 4(q-2, q, 2)$ graphs.

In view of the remark on case (2)(ii) in Proposition 1, we have the following result.

Theorem 3. Let $\Gamma$ be a Krein graph of diameter 4. Then one of the following assertions holds:

(1) $\bar{\Gamma}$ is a Smith graph without triangles, and $\Gamma$ has the intersection array $\{k, k-\mu,(r-1) \mu, 1 ; 1, \mu, k-1$, $k\}$, where $K=t^{3}+3 t^{2}+t$ and $r \mu=t(t+1)$.

(2) $\bar{\Gamma}$ is a Smith graph with $a_{1}>0$ and nonprincipal eigenvalues $t$ and $s$; and the nonprincipal eigenvalues of the constituents of $\bar{\Gamma}$ are equal to $t, s_{1}=\frac{t^{2}+2 t+s}{2}, t$, $s_{2}=\frac{s-t^{2}}{2}$, and either

(i) $t=q-2, \Gamma$ is a tight graph $\mathrm{AT} 4(q-2, q, r)(2<$ $r \leq q-1)$ with the intersection array $\left\{q\left(q^{2}-2\right),\left(q^{2}-\right.\right.$ 1) $(q-1), \frac{(r-1) \cdot 2 q(q-1)}{r}, 1 ; 1, \frac{2 q(q-1)}{r},\left(q^{2}-\right.$ $\left.1)(q-1), q\left(q^{2}-2\right)\right\}$ and, for any vertex a of $\Gamma$, the $s u b$ graph $\Gamma_{2}(a)$ is an antipodal distance-regular graph of diameter 4 with the intersection array $\left\{(q-2) q^{2},(q-\right.$ $1)^{3}, \frac{2(r-1)(q-1)(q-2)}{r}, 1 ; 1, \frac{2(q-1)(q-2)}{r},(q-$ $\left.1)^{3},(q-2) q^{2}\right\}$ or

(ii) $s$ and t are even and, for $q=t+2$ and $u=\frac{s+q^{2}}{2}$, we have $\sqrt{q} \leq u<q-1, q(q-1)-u$ divides $(q-2)^{2}(q-$ $1)^{2} q$, and the expression $4 u^{4}(q-2)^{2}-4 u^{3}(q-1)\left(q^{3}-\right.$ $\left.q^{2}-6 q+10\right)+u^{2}(q-1)^{2}\left(q^{4}+6 q^{3}-15 q^{2}+12 q+20\right)-$ $2 u(q-1)^{3}\left(q(q+1)\left(q^{2}+4\right)\right)+q^{2}(q-1)^{4}(q+2)^{2}$ is a square.

The following result holds for AT4-graphs.
Theorem 4. Let $\Gamma$ be an $\mathrm{AT} 4(p, q, r)$-graph, $u$ be $a$ vertex of $\Gamma$, and $\Delta=[u]$. Then the following assertions hold:

(1) If $p=\alpha q$, then $\Delta$ is a pseudogeometric graph for $p G_{\alpha}(\alpha(q+1), q-1), \alpha+1$ divides $2\left(q^{2}-1\right), \alpha+q$ divides $q\left(q^{2}-1\right)\left(q^{2}+g-1\right)(q+2), \frac{q^{3} \alpha(\alpha+1)}{r}$ is even, $r(p+1) \leq q(p+q)$, and $r$ divides $q(\alpha+1)$.

(2) If $\Delta$ is a graph without triangles, then $\Gamma$ is a Conway-Smith graph or the first Soicher graph with $(p, q, r)=$ $(2,4,3)$.

(3) If $q=p+2$, then

(i) $\frac{2 p(p+1)(p+2)}{r}$ is even, $2<r<p+2, r$ divides $2(p+1)$, and $\Gamma$ is a graph with the intersection array $\left\{(p+1)(p+2)^{2},(p+3)(p+1)^{2}, \frac{(r-1) \cdot 2(p+1)(p+2)}{r} ;\right.$ $\left.1,1, \frac{2(p+1)(p+2)}{r},(p+3)(p+1)^{2},(p+1)(p+2)^{2}\right\}$.

(ii) The antipodal quotient $\bar{\Gamma}$ is a graph with parameters $\left(\frac{(p+1)^{2}(p+4)^{2}}{2},(p+2)\left(p^{2}+4 p+2, p(p+3)\right.\right.$, $2(p+1)(p+2))$ and eigenvalues $p,-\left(p^{2}+4 p+4\right)$.

(iii) The second neighborhood of a vertex in $\bar{\Gamma}$ is a strongly regular graph with parameters $\left((p+1)(p+3)\left(p^{2}+\right.\right.$ $\left.4 p+2), p(p+2)^{2}, p^{2}+p-2,2 p(p+1)\right)$ and eigenvalues $p,-\left(p^{2}+2 p+2\right)$ that has a distance-regular $r$-covering with the intersection array $\left\{p(p+2)^{2},(p+1)^{3}\right.$, $\left.\frac{2(r-1) p(p+1)}{r}, 1 ; 1, \frac{2 p(p+1)}{r},(p+1)^{3}, p(p+2)^{2}\right\}$.

(4) If $p \leq 4$, then either

(i) $\Gamma$ is a Conway-Smith graph or a Soicher graph with $(p, q, r)=(2,4,3)$,

(ii) $\Gamma$ is a $3 O_{6}^{-}$(3)-graph or $p=3, q=5, r=4$, and $\Gamma$ has the intersection array $\{115,96,30,1 ; 1,10,96$, $115\}$, while $\Gamma_{2}(u)$ has the intersection array $\{75,64,18$, $1 ; 1,6,64,75\}$, or

(iii) $\Gamma$ is a half-8-cube or $(p, q, r)=(4,4,2)$ and $\Gamma$ has the intersection array $\{96,75,16,1 ; 1,16,75,96\}$, or $(p, q, r)=(4,6,5)$ and $\Gamma$ is a graph with the intersection array $\{204,175,48,1 ; 1,12,175,204\}$ and $\Gamma_{2}(u)$ is a graph with the intersection array $\{144,125,32,1 ; 1$, $8,125,144\}$.

(5) If $q \leq 4$, then either 
(i) $\Gamma$ is a Johnson graph $J(8,4)$, a half-8-cube, or a Conway-Smith graph,

(ii) $\Gamma$ is a $3 O_{6}^{-}(3)$-graph, $3 O_{7}(3)$-graph, or $(p, q, r)=$ $(p, 3,2)$, $\Gamma$ is a graph with the intersection array $\{3(4 p+3)$, $\left.8(p+1), \frac{3(p+3)}{2}, 1 ; 1, \frac{3(p+3)}{2}, 8(p+1), 3(4 p+3)\right\}$ and $p=9,21$, or

(iii) $\Gamma$ is a half-8-cube, the second Meixner graph, the first Soicher graph, or $r=3, \Gamma$ is a graph with the intersection array $\left\{4(5 p+4), 15(p+1), \frac{8(p+4)}{3}, 1 ; 1\right.$, $\left.\frac{8(p+4)}{3}, 15(p+1), 4(5 p+4)\right\}$ and $p=8,20,44,56$, 116 , or $r=2, \Gamma$ is a graph with the intersection array $\{4(5 p+4), 15(p+1), 4(p+4), 1 ; 1,4(p+4), 15(p+1)$, $4(5 p+4)\}$ and $p=8,9,20,21,44,56,116$.

Let $n+1=r u, \Gamma$ be a Krein graph of diameter 3 with the intersection array $\left\{n^{3},(r-1) u\left(n^{2}-1\right), 1 ; 1\right.$, $\left.u\left(n^{2}-1\right), n^{3}\right\}, a$ be a vertex of $\Gamma$, and $\Delta=[a]$. It was proved in [6, Lemma 5.3] that $\Delta$ is a strongly regular graph with parameters $V=n^{3}, k=(n-$ 1) $\left(\frac{(n+1)^{2}}{r}-n\right), \lambda=r\left(\frac{n+1}{r}-1\right)^{3}+r-3, \mu=$ $\left(\frac{n+1}{r}-1\right)\left(\frac{(n+1)^{2}}{r}-n\right)$ and with eigenvalues $k, n-$ $\frac{n+1}{r}, n-\frac{(n+1)^{2}}{r}$. Therefore, $\Delta$ is a pseudogeometric graph for $p G_{u-1}\left(r u-2, r u^{2}-r u\right)$ with eigenvalues $k=$ $(r u-2)\left(r u^{2}-r u+1\right), r u-1-u, r u-1-r u^{2}$. The integer-valuedness assumption, i.e., $(u-1)\left(r u^{2}-u\right)$ divides $(r u-2)(r u-1)\left(r u^{2}-r u\right)\left(r u^{2}-r u+1\right)$, holds true.

Lemma 1. If $n$ is a power of a prime number, then the following assertions hold:

(1) If $r=\frac{n+1}{2}$, then there exists a graph $\Gamma$ that is locally a $G Q(n-1, n+1)$-graph.

(2) There exist pseudogeometric graphs for $p G_{2}(4$, 12), $p G_{3}(6,24), p G_{2}(7,18), p G_{4}(8,40), p G_{2}(10,24)$, $p G_{3}(10,36), p G_{5}(10,60)$, and $p G_{6}(12,84)$.

Proof. If $n$ is a power of a prime number, then, in view of the remark presented after Lemma 5.2 in [6], $\Gamma$ exists; therefore, $\Delta$ exists as well. If $r=\frac{n+1}{2}$, in view of [7], there exists a graph $\Gamma$ that is locally a $G Q(n-1$, $n+1)$-graph.

\section{DOKLADY MATHEMATICS Vol. $88 \quad$ No. 2013}

For graphs with $n \leq 13$, pseudogeometric graphs exist for $G Q(4,6)$ and $p G_{2}(4,12)(n=5)$; for $G Q(6,8)$ and $p G_{3}(6,24)(n=7)$; for $p G_{2}(7,18)(n=8)$; for $G Q(8$, $10)$ and $p G_{4}(8,40)(n=9)$; for $G Q(10,12), p G_{2}(10,24)$, $p G_{3}(10,36)$, and $p G_{5}(10,60)(n=11)$; and for $G Q(12$, $14)$ and $p G_{6}(12,84)(n=13)$. Lemma 1 and Theorem 1 are proved.

\section{Lemma 2. $\operatorname{AT} 4(q-2, q, 2)$-graphs do not exist.}

Proof. Let $\Gamma$ be an $\operatorname{AT} 4(q-2, q, 2)$-graph with the intersection array $\left\{q\left(q^{2}-2\right),\left(q^{2}-1\right)(q-1), q(q-1)\right.$, $\left.1 ; 1, q(q-1),\left(q^{2}-1\right)(q-1), q\left(q^{2}-2\right)\right\}$. Then the neighborhood of a vertex $u$ in $\Gamma$ is a strongly regular graph with parameters $\left(q^{3}-2 q,(q+1)(q-2), q-4\right.$, $q-2$ ) and, by Theorem 5.8 in [4], for any two vertices $y$ and $z$ separated by a distance at most 2 in $\Gamma_{2}(u)$, we have $|[u] \cap[y] \cap[z]|=q-1$. From this, for any two vertices $w, w^{\prime} \in[u]$, it is true that $\left|[w] \cap\left[w^{\prime}\right] \cap \Gamma_{2}(u)\right|=$ $q^{2}-2 q+1$.

Thus, the pair $\left([u], \Gamma_{2}(u)\right)$ is a quasi-symmetric $\left(q\left(q^{2}-2\right),\left(q^{2}-2\right)\left(q^{2}-1\right),\left(q^{2}-1\right)(q-1), q(q-1)\right.$, $\left.q^{2}-2 q+1\right)$-scheme in which any two blocks intersect in 0 or $q-1$ points.

By [8, Theorem 5.3], a block graph of a scheme is strongly regular with eigenvalues $\frac{(r-1) k-x b-y}{y-x}=$ $q^{2}\left(q^{2}-q-1\right)-1, q^{2}-2 q,-q$ and parameters $\left(\left(q^{2}-\right.\right.$ 2) $\left(q^{2}-1\right), q^{2}\left(q^{2}-q-1\right)-1, q^{4}-2 q^{3}+2 q^{2}-3 q-1$, $\left.q^{4}-2 q^{3}+q^{2}-1\right)$. The multiplicity of the eigenvalue $q^{2}-2 q$ is $\frac{\left.q^{2}\left(q^{2}-q-1\right)-1\right)\left(q^{2}\left(q^{2}-q-1\right)+q-1\right)}{q\left(q^{4}-2 q^{3}+q^{2}-1\right)}$, which contradicts the fact that the numerator of the fraction is not divided by $q$. The lemma and Theorem 2 are proved.

Lemma 3. Let $\Gamma$ be an $\mathrm{AT} 4(p, q, r)$-graph, $u$ be a vertex of $\Gamma$, and $\Delta=[u]$. If $p=3$, then either $q=r=3$ and $\Gamma$ is a $3 O_{6}^{-}(3)$-graph or $q=5, r=4, \Delta$ has parameters $(115,18,1,3)$, and $\Gamma$ has the intersection array $\{115$, $96,30,1 ; 1,10,96,115\}$.

Proof. Let $p=3$. Then $\Delta$ has parameters $\left(4 q^{2}+3 q\right.$, $3(q+1), 6-q, 3)$. Furthermore, $q+3$ divides $q^{2}\left(q^{2}-\right.$ $1)$ and $q^{2}+3$ divides $q^{2}\left(q^{2}-1\right)\left(q^{2}+q-1\right)(q+2)$.

If $q=3$, then $\Delta$ has parameters $(45,12,3,3)$. In this case, $r=3$ and $\Gamma$ is a $3 O_{6}^{-}(3)$-graph.

If $q=5$, then $\Delta$ has parameters $(115,18,1,3)$ and eigenvalues 3 and -5 . By Lemma 2, the case $r=2$ is not possible. Therefore, $r=4$ and $\Gamma$ has the intersection array $\{115,96,30,1 ; 1,10,96,115\}$.

If $q=6$, then $\Delta$ has parameters $(162,21,0,3)$ and eigenvalues 3 and -6 . In this case, $q^{2}+3=39$ does not divide $q^{2}\left(q^{2}-1\right)\left(q^{2}+q-1\right)(q+2)$.

The cases $p=4$ and $q=3,4$ are treated in a similar manner. 


\section{ACKNOWLEDGMENTS}

This work was supported by the Russian Foundation for Basic Research (project no. 12-01-00012 and joint project no. 12-01-91155 with the National Science Fund of China), by the Branch of Mathematics of the Russian Academy of Sciences (project no. 12T-1-1003), and by the Ural Branch of the Russian Academy of Sciences jointly with the Siberian Branch of the Russian Academy of Sciences (project no. 12-S1-1018) and with the National Academy of Sciences of Belarus (project no. 12-S-1-1009).

\section{REFERENCES}

1. A. E. Brouwer, A. M. Cohen, and A. Neumaier, Distance-Regular Graphs (Springer-Verlag, Berlin, 1989).
2. A. Jurisic and J. Koolen, Discrete Math. 244, 181-202 (2002).

3. I. P. Belousov, A. A. Makhnev, and M. S. Nirova, Dokl. Math. 86, 816-819 (2012).

4. A. Jurisic, Discrete Math. 264 (1-3), 127-148 (2003).

5. A. Jurisic and J. Koolen, J. Comb. Theory 118, 842$852(2011)$.

6. C. D. Godsil, Austral. J. Comb. 6, 245-255 (1992).

7. P. Cameron, Discrete Math. 97, 83-92 (1991).

8. P. J. Cameron and J. van Lint, Graphs, Codes, and Designs (Cambridge Univ. Press, Cambridge, 1980).

Translated by I. Ruzanova 